\title{
Fuzzy clustering and Whale-based neural network to food recognition and calorie estimation for daily dietary assessment
}

\author{
W R SAM EMMANUEL and S JASMINE MINIJA* \\ Department of Computer Science, Nesamony Memorial Christian College, Marthandam, Affiliated to \\ Manonmaniam Sundaranar University, Tirunelveli 627 012, India \\ e-mail: minijakenson@gmail.com
}

MS received 28 September 2017; revised 26 February 2018; accepted 26 February 2018; published online 14 May 2018

\begin{abstract}
The calorie value of the food items taken by the person in everyday life needs to be monitored to reduce the risk of obesity, heart problems, and diabetes, etc. The calorie estimator in the existing models has reduced accuracy since the calorie value of each food varies with mass. This paper introduces a dietary assessment system based on the proposed Cauchy, Generalized T-Student, and Wavelet kernel based Wu-and-Li Index Fuzzy clustering (CSW-WLIFC) based segmentation and the proposed Whale Levenberg Marquardt Neural Network (WLM-NN) classifier. The proposed CSW-WLIFC based segmentation segments the image based on the existing WLI-FC algorithm. A novel CSW based kernel function is utilized in the segmentation process. Feature vectors such as color, shape, and texture are extracted from the segmented image. The Neural Network is trained with the Whale-Levenberg Marquardt (WLM) model to recognize each food item from the tray image. The proposed calorie estimator calculates the calorie value of each food item. From the simulation results, it is evident that the proposed model has the improved performance than the existing models with the values of $0.999,0.9643,0.9627$, and 0.0184 for the segmentation accuracy, macro average accuracy, standard accuracy, mean square error, respectively.
\end{abstract}

Keywords. Dietary assessment; CSW-WLIFC algorithm; Whale-Levenberg Marquardt; segmentation accuracy.

\section{Introduction}

A Recent report by the World Health Organisation (WHO) suggests that the tremendous increase in various diseases such as heart problems, lung infection, cancer, and diabetes occur due to the wrong food intake and obesity to various activities. These diseases are occurred due to the inefficient or the excessive intake of the food items in the daily life [1]. Food obesity has tremendously increased due to the wrong intake of the food items. Hence, there is a need for the dietary assessment system [2-4] to estimate the calorie intake by each person [5]. The major challenge in the assessment of the calorie value is the recognition of the food items from the group of food images. The food images are usually captured as the food present in the tray. The food items need to be separated from the food and the non-food category to estimate the precise calorie value [6]. More researchers concentrate on grouping the similar food items in the image and then estimating its calorie value. Accurate assessment of the food item from the food

*For correspondence image is a major challenge in the dietary assessment system [7].

The dietary assessment system [8-12] can be developed by recording the set of food items consumed by the individual with the use of the mobile phone. The nutrient intake for the particular day can be obtained through this method. But the above method is time-consuming and erroneous, since the manual recording of the everyday activity may be misleading. To avoid this problem [13], image recognition comes in handy. The food items are recorded as the food images and are subjected to processing for the calorie estimation. The dietary assessment based on the image recognition is based on three steps. They are; (i) segmentation, (ii) feature extraction and (iii) food recognition [14]. The image segmentation process segments each image by locating the objects and boundaries in images. The paper [15] has segmented the food items by placing the similar ingredient in the same segment. The other segmentation algorithms used are adaptive thresholding [16], connected component analysis [17], etc.

Features such as color, texture, and shape are the major factors for the feature extraction process. The feature extraction is done in two ways. They are global and local feature extraction. Average color features and Gabor 
Sādhanā (2018) 43:78

features are used in [17] while in [18], food items are described by a bag of SIFT features. The texture feature for the recognition of the food item uses the LBP, LVP, etc. and finally the shape features are also obtained [19]. Once the features are attained, then the food items are classified by the competent classifiers. Such classifiers are named as Support Vector Machine (SVM) [7], Extreme Learning Machine (ELM) [1], etc. The nutritional value of the food item is not known by most of the person [20]. Hence, the user finds it difficult to estimate the calorie value of the food item [21].

This paper proposes a dietary assessment system with calorie calculator. The proposed system finds the calorie value of each food items from the image in three steps. They are segmentation, feature extraction, and food recognition. The images from the food database are segmented to various clusters using the proposed Cauchy, Generalized T-Student, and Wavelet based Wu-and-Li Index Fuzzy clustering(CSW-WLIFC). The proposed CSW-WLIFC algorithm performs the clustering of food images and hence separates each food item from the food image. The proposed CSW-WLIFC algorithm uses a novel CSW based kernel function to perform the segmentation process. The features from the segmented food images are extracted with the use of the feature extraction process. The features, such as color, shape, and local vector pattern (LVP) are extracted from the food image during the feature extraction process. The proposed Whale Levenberg Marquardt Neural Network (WLM-NN) classifier recognizes each food item with the use of the extracted features. The NN model gets trained with the proposed WLM model for better recognition of the food items in the image. Finally, the calorie value of each food item is calculated with the calorie calculator.

The major contribution of this research work is to develop the CSW-WLIFC mechanism based on the CSW based kernel and the existing WLI-FC algorithm for the segmentation of the food images. The CSW based kernel function is newly proposed by considering the kernel functions, such as Cauchy, Generalized T-Student, and Wavelet. Also, WLM-NN classifier is proposed to train the $\mathrm{NN}$ with the existing LM model and the WOA, which also performs classification of the food items from the image. At last, the calorie calculator is designed to estimate the calorie value of the recognized food items.

The remaining part of this paper is organized as follows: Section 2 surveys various existing works based on the dietary assessment. It also discusses the various challenges of the design process. Section 3 briefly explains the proposed dietary assessment system based on the kernel-based WLI fuzzy clustering and the WNN network. Section 4 provides the simulation results of the proposed system, and it compares the performance with the existing models. Section 5 concludes the paper with the summary and the future work.

\section{Literature review}

In this section, various literature has been analyzed for the efficient classification of the food images from the database. Various segmentation and the classification processes used in the related works have been discussed.

Fengqing Zhu et al [7] developed the dietary assessment system which automatically identified the food items from the input image. The food items are segmented using the multiple hypothesis methods. Then, the food features were extracted with the aids of global and local feature extraction method. Consequently, the extracted features were classified by the KNN and SVM classifier which can help to measure the calorie value efficiently. Then, the food recognition system based on a group classification was demonstrated in [1]. Niki Martinel et al [1] provided a system capable of automatically choosing the optimal features for food recognition using Extreme Learning Machine (ELM). Then, the structural SVM was used to filter out the irrelevant features for the final ranking of possible matches of food types. The automatic food recognition system was explained by Marios Anthimopoulos et al [22] using Bagof-Features. The local features of the food items were obtained and exploited the SIFT descriptor for the HSV color space. After the feature vector was obtained, the SVM classified the food items significantly, thus attained the higher accuracy. To improve the classification performance, Hokuto Kagaya et al [13] deliberated the food detection and recognition using convolution neural network. The CNN was defined as the efficient deep learning technique which performed through the parameter optimization. Thus, the CNN [23] showed the better performance rather than SVM method. In addition, the calorie value of every food item was measured in [21]. Normally, people might not be aware of the calorie value of food items to maintain the diet. Thus, calorie values were presented in the dietary assessment system where the person acquired the dietary food intake. Marios Anthimopoulos et al [14] explained the segmentation and recognition of multi-food meal images. The food items were segmented using the mean-shift algorithm. Once the texture descriptor obtained the feature, the SVM classifier was employed to identify the food images. To resolve the issue of paperbased food recording, MdHafizur Rahman et al [6] improved the food recognition accuracy in mobile phonebased dietary assessment system. The mobile phone camera captured the food images considered the input image for the dietary assessment system. The texture feature of food items was extracted by the Gabor filters and scale invariance. The resultant feature was classified efficiently to recognize the food items.

Mogale et al [24] have proposed an integrated multiobjective, multi-modal and multi-period mathematical model for grain silo location-allocation problem with Dwell time to support the decision-making process of 
Table 1. The summary of related works.

\begin{tabular}{|c|c|c|c|c|}
\hline Study & $\begin{array}{l}\text { Single/ } \\
\text { Multi-period }\end{array}$ & $\begin{array}{l}\text { Single/multi- } \\
\text { model }\end{array}$ & Model & Objectives \\
\hline Fengqing Zhu et al [7] & Single & Single & $\begin{array}{c}\text { linear, quasi-linear, and non- } \\
\text { linear element } \\
\text { kernels }\end{array}$ & $\begin{array}{c}\text { To address the variance problem of food } \\
\text { appearances }\end{array}$ \\
\hline Niki Martinel et al[1] & Single & Single & Linear Programming & $\begin{array}{c}\text { To automatically } \\
\text { identify and locate food in a variety of images } \\
\text { captured } \\
\text { during controlled and natural eating events }\end{array}$ \\
\hline $\begin{array}{l}\text { Marios M. } \\
\text { Anthimopoulos et al } \\
\text { [22] }\end{array}$ & Single & Single & Linear Programming & $\begin{array}{c}\text { To provide an accurate account of daily food } \\
\text { and nutrient } \\
\text { Intake. }\end{array}$ \\
\hline $\begin{array}{l}\text { Hokuto Kagaya et al } \\
\text { [13] }\end{array}$ & Single & Single & Texture Feature & To detect and recognize food images. \\
\hline $\begin{array}{l}\text { Marios Anthimopoulos } \\
\quad \text { et al }[14]\end{array}$ & Multi & Multi & Linear and non-linear & $\begin{array}{l}\text { Automatic segmentation and recognition of } \\
\text { multi-food } \\
\text { Images. }\end{array}$ \\
\hline $\begin{array}{l}\text { MdHafizur Rahman } \\
\text { et al [6] }\end{array}$ & Multi & Multi & Linear and non-linear & $\begin{array}{l}\text { To extract features that are invariant to the } \\
\text { scale and rotation of the texture in an image }\end{array}$ \\
\hline Mogale et al [24] & Multi & Multi & Non-linear programming & $\begin{array}{c}\text { Solve grain silo location-allocation problem } \\
\text { with Dwell time to support } \\
\text { the decision-making process of GOI. }\end{array}$ \\
\hline Mogale et al [25] & Multi & Multi & Non-linear & $\begin{array}{c}\text { To minimize the bulk food grain transportation } \\
\text { inventory holding, and } \\
\text { operational cost. }\end{array}$ \\
\hline
\end{tabular}

Government of India (GOI). In this method, two objectives, such as minimization of total supply chain network cost and total lead time are simultaneously optimized by two Pareto based multi-objective algorithms with calibrated parameters. The major advantage of this method is that the total lead time decreases due to the establishment of new base and field silos. The high complexity is the drawback of this work. Mogale et al [25] have presented a Mixed Integer Non-Linear Programming (MINLP) model for planning the movement and storage of food grain from surplus states to deficit states by taking into consideration of vehicle capacity constraints, demand satisfaction, silo capacity, and the seasonal procurement. The main objective of this work is to minimize the operational cost, inventory holding, and bulk food grain transportation. This method was computationally complex in nature due to nonlinearity, the presence of numerous integer and binary variables along with a huge number of constraints.

Table 1 shows the summary of the related works based on some characteristics, such as single/multiple period, single/multiple model, model, and objectives.

\subsection{Challenges}

The dietary assessment system for the recognition of food images has the following challenges,
- The human interaction in the collection of the information about the nutrient value of the food item is a tedious task.

- The food items available has a different texture, color and shape formation and hence the feature extraction is considered as another problem.

- The food items have different illumination and viewpoint. The non-rigid deformations and intra-class variability in the food items make the food recognition task to be difficult.

- The SVM classifier for the image recognition is more suitable for the binary classification only.

- The image taken from the database for the simulation contains the images of the food items present in the plates over the dining table. The table with the food items contains other objects such as clothes, cell phones, spoons, plates, and fork. Hence, the segmentation of the food item from the images is a more challenging task.

- The food images from the database contain a variety of food rather than the single food. Each food item has different calorie value from the other. Hence, the feature extraction of each segmented food item should be more precise.

- Since the images contain many food items, the proposed classifier needs larger training data.

- The classification method needs many computing resources, mostly for the ingredient detection part [7]. 
- The segmentation of the food items is difficult due to faint boundary edges that camouflage the food item [1].

- The methods proposed in $[6,13]$ have high computational cost.

- It is very challenging to extract the major diameter and minor diameter of a spherical object from a single view image in perspective projection [22].

- The method in [14] needs further improvements in the segmentation process.

- The high complexity is the drawback as given in [24] and [25].

\section{Proposed methodology}

This paper introduces the dietary assessment system through the efficient identification of the calorie values of each food items from the food image. The block diagram of the proposed dietary assessment system is shown in figure 1 . The food database contains various food or the meal images consumed by the person in their daily life. The images in food database are preprocessed for the better processing. The food images contain the combination of bread, cereals, fruits, and vegetables, etc. Hence, each food item in the food image needs to be classified before identification of the food items. The proposed CSW-WLIFC algorithm performs the segmentation of each food items from the food images. After the segmentation of the food images to various clusters, the features are extracted from the segmented image. The feature extraction extracts the features, such as shape, color, and texture from the image. The extracted features from the images are then subjected to the classification for the identification of the foods. The proposed WLM-NN classifier performs to train the neural network (NN) with the existing LM model and the Whale Optimization Algorithm (WOA), which also performs classification of the food items from the image. The dietary assessment system finds the calorie value of the recognized food items obtained from the WLM-NN classifier.

Consider a food database $D$ with $N$ number of food images. Each food images have various food items. The food image database can be expressed by the equation (1).

$$
D=\left\{I_{f}\right\} ; \quad 1 \leq f \leq N
$$

The images in the food database have the size of $K \times L$. Pixels in the food image are represented by $(u, v)$. The food image $I_{f}$ can be expressed by the equation (2).

$$
I_{f}=\left\{I_{f}(u, v)\right\} ; \quad 1 \leq u \leq K ; \quad 1 \leq v \leq L
$$

\subsection{Preprocessing}

The initial step in the design of the dietary assessment system is preprocessing. The tray images from the food database are preprocessed before the segmentation process. The preprocessing is done in two steps: Resizing and ROI (Region of Interest) extraction.

Resizing: The tray images from the dataset are resized to make it more suitable for the segmentation process. The original image contains the tray image present in the food table. Hence, for the better segmentation process, the image section with the tray is extracted from the original image.

ROI extraction: The next step in the preprocessing is the ROI extraction. The original image from the dataset contains more than one food items present in the different tray. Besides the food items, the image also contains spoon, fork,



Figure 1. Block diagram of the proposed methodology. 
mobile phones, and purse, etc. also may be available with the images. Hence, in the ROI extraction process, the food item is extracted from the resized image.

\subsection{Segmentation of the food image}

The food images from the database contain the images of the meals with the combination of the various food items. Every food items in the food image have varying masses and calorie value. Hence, each food items in the food images need to be segmented before extracting the features. The CSW-WLIFC algorithm performs the segmentation of the food images. The CSW-WLIFC algorithm uses various kernel functions along with the existing WLI-FC algorithm [26-29] for the segmentation. Figure 2 shows the block diagram of the segmentation process with the CSW-WLIFC algorithm. The segmentation of the food items in the images can be achieved through the clustering of the food items in the image $I_{f}$.

3.2a Construction of the data matrix: The initial step in the segmentation process is the construction of the data matrix $M_{i j}^{f}$ from the image $I_{f}$. The food image $I_{f}$ has the size of $K \times L$. The data matrix of the food image can be constructed by considering the illumination pixels of the food image $I_{f}$. Each pixel in the food image contains nine illumination features. Thus the data matrix for the image $I_{f}$ contains $K \times L$ rows and nine columns. The size of the data matrix is $K \times L \times 9$. The term $M_{i j}^{f}$ indicates the data matrix of the image $I_{f}$. The term $i$ indicates the rows in the data matrix and $j$ indicates the columns in the data matrix. The value of $i$ varies between 1 and $J$ and the value of $j$ varies between 1 and 9 , where $J=K \times L$. The pixels of the image $I_{f}$ is present in the first column of the data matrix. The remaining eight columns of the data matrix contain the illumination features of the image.

\section{2b Algorithmic procedure of CSW-WLIFC:}

\section{Step 1: Initialization}

Clustering [30-32] of the data matrix $M_{i j}^{f}$ allows the segmentation of the image $I_{f}$. The initial step of CSWWLIFC algorithm is the initialization of the required number of clusters to perform the clustering. The total cluster size for the clustering is chosen based on the total food items in the food image. The total number of clusters in the image is indicated as $A$. The cluster size varies as $1 \leq a \leq A$. Each cluster $A_{a}$ in the image has the centroid point. The centroid for the formation of the clusters is chosen at random. The centroids of the cluster vary between 1 and A. Consider a random cluster $B_{a}$ in the $a$ th cluster.

Step 2: Distance calculation using CSW-based kernel The clusters within the image are formed by choosing the random cluster centroid point in the image. The term $B_{a}$ indicates the centroid of the cluster $A_{a}$. The pixels in the data matrix can be grouped into the cluster $A_{a}$ by calculating the distance between the pixels and the centroid point $B_{a}$. The equation (3) expresses the distance between the pixels of the data matrix and the centroid point.

$$
E_{i a}^{f}=F\left(M_{i}^{f}, B_{a}\right)
$$

Where $E_{i a}^{f}$ indicates the distance between the pixels of the data matrix $M_{i}^{f}$ and the centroid $B_{a}$. This paper proposes the Cauchy, Generalized T-Student, and Wavelet (CSW) based kernel distance function based on the kernel functions, such as Cauchy kernel function, Generalized T-



Figure 2. Segmentation using the proposed CSW-WLIFC algorithm. 
Student kernel function, and the Wavelet kernel function. The term $F^{C}$ indicates the Cauchy kernel function. The Cauchy kernel has more long-range influence and sensitivity on the high dimensional space. The term $F^{S}$ indicates the Generalized T-Student kernel function. The Generalized T-Student kernel function has a positive semidefinite kernel matrix. It allows the extraction of the global and the local features from the images. The term $F^{W}$ indicates the Wavelet kernel function. The wavelet kernel was formed based on the wavelet theory. The term $F\left(M_{i}^{f}, B_{a}\right)$ indicates the CSW-based kernel distance between the pixels of the data matrix $M_{i}^{f}$ and the random centroid point. The equation (4) expresses the general formula of the newly formed CSW-based kernel distance. The CSW-based kernel distance between the matrix and the centroid $B_{a}$ defines the sum of the Cauchy kernel $F^{C}\left(M_{i}^{f}, B_{a}\right)$, Generalized T-Student kernel $F^{S}\left(M_{i}^{f}, B_{a}\right)$, and the Wavelet kernel $F^{W}\left(M_{i}^{f}, B_{a}\right)$.

$$
F\left(M_{i}^{f}, B_{a}\right)=\left\{F^{C}\left(M_{i}^{f}, B_{a}\right)+F^{S}\left(M_{i}^{f}, B_{a}\right)+F^{W}\left(M_{i}^{f}, B_{a}\right)\right\}
$$

The CSW-based kernel distance based on the Cauchy kernel $F^{C}\left(M_{i}^{f}, B_{a}\right)$ between the data matrix and the centroid $B_{a}$ is given by the equation (5). The term $\sigma$ indicates the scale parameter in the Cauchy function.

$$
F^{C}\left(M_{i}^{f}, B_{a}\right)=\frac{1}{1+\frac{\left\|M_{i}^{f}-B_{a}\right\|^{2}}{\sigma^{2}}}
$$

The CSW-based kernel distance based on the Generalized T-Student kernel $F^{S}\left(M_{i}^{f}, B_{a}\right)$ is given by the equation (6).

$$
F^{S}\left(M_{i}^{f}, B_{a}\right)=\frac{1}{1+\left\|M_{i}^{f}-B_{a}\right\|^{e_{2}}}
$$

The CSW-based kernel distance based on the Wavelet kernel is given by the equation (7). The term $\zeta\left(M_{i}^{f}\right)$ indicates the mother wavelet function and $e_{3}$ indicates the Wavelet dilation. The mother wavelet function of the data matrix $\zeta\left(M_{i}^{f}\right)$ is indicated by the equation (8). The term $J$ indicates the size of the row of the data matrix, i.e., $J=K \times L$.

$$
\begin{gathered}
F^{W}\left(M_{i}^{f}, B_{a}\right)=\prod_{1=1}^{J} \zeta\left(\frac{M_{i}^{f}-B_{a}}{e_{3}}\right) \\
\zeta\left(M_{i}^{f}\right)=\cos \left(1.75 M_{i}^{f}\right) \exp \left(\frac{-M_{i}^{f^{2}}}{2}\right)
\end{gathered}
$$

Step 3: Clustering of the image based on the membership function

The pixels in the image are clustered by grouping the pixels nearer to each other on the chosen centroid point. Hence, the membership function with the minimum objective is defined. The membership function chooses the pixels around the cluster with the minimum distance from the centroid point. The membership function based on fuzzy objective gets modified based on the proposed CSW based kernel functions $F^{C}, F^{S}$, and $F^{W}$. Equation (9) indicates the membership function of the CSW-WLIFC algorithm.

$$
G_{i a}^{f}=\frac{1}{\sum_{k=1}^{A}\left[\frac{F\left(M_{i}^{f}, B_{a}\right)}{F\left(M_{i}^{f}, B_{k}\right)}\right]^{\frac{2}{e-1}}}
$$

The term $F\left(M_{i}^{f}, B_{k}\right)$ indicates the CSW-based kernel distance between the pixels of the data matrix and the newly formed centroid point $B_{k}$. The value $e$ indicates the integer, and it varies between $1 \leq e \leq \infty$. The CSW-based kernel distance to the new centroid of the cluster is given as, $F\left(M_{i}^{f}, B_{k}\right)$. The equation (10) expresses the CSWbased kernel distance for the new centroid.

$$
F\left(M_{i}^{f}, B_{k}\right)=\left\{F^{C}\left(M_{i}^{f}, B_{k}\right)+F^{S}\left(M_{i}^{f}, B_{k}\right)+F^{W}\left(M_{i}^{f}, B_{k}\right)\right\}
$$

Where, $F^{C}\left(M_{i}^{f}, B_{k}\right), F^{S}\left(M_{i}^{f}, B_{k}\right)$ and $F^{W}\left(M_{i}^{f}, B_{k}\right)$ indicates the CSW-based kernel distance between the data matrix and the centroid $B_{k}$ based on the Cauchy, Generalized T-Student, and the Wavelet kernel, respectively. The generalized objective function derived in the equation (4) allows the clustering of the pixels in the image based on the defined CSW-based kernel distance. The centroid of the cluster gets updated based on the defined generalized objective function. The membership function for the proposed CSW-WLIFC algorithm uses the newly formed CSW-based kernel distance for evaluating the distance between the pixels and the centroid. The updated centroid equation is given by the equation (11).

$$
B_{a}=\frac{\sum_{i-1}^{J}\left(G_{i a}^{f}\right)^{e} * M_{i}^{f}}{\sum_{i-1}^{J}\left(G_{i a}^{f}\right)^{e}}
$$

Step 4: Finding the validity of the cluster through the cluster validity index (CVI)

The cluster formed by the use of the objective function and the new centroid can be declared valid if the following condition is satisfied. The equation (12) expresses the mathematical form of the CVI. The CVI finds the validity of the cluster $A_{a}$ with the use of the fuzzy compactness of the cluster $Y_{p}$ and the separation between the clusters $Y_{q}$. 
The proposed algorithm selects the cluster with the minimum CVI value for better segmentation of the pixels in the image.

$$
X\left(A_{a}\right)=\frac{Y_{p}}{2 * Y_{q}}
$$

The fuzzy compactness $Y_{p}$ of the cluster $A_{a}$ is expressed in the equation (13). The fuzzy compactness includes the CSW-based kernel distance of the data matrix $M_{i}^{f}$ and the centroid $B_{a}$ of the cluster. The fuzzy compactness value should be minimum for the better segmentation process.

$$
Y_{p}=\sum_{a=1}^{A}\left[\frac{\sum_{i-1}^{J}\left(G_{i a}^{f}\right)^{2} F\left(M_{i}^{f}, B_{a}\right)^{2}}{\sum_{i-1}^{J}\left(G_{i a}^{f}\right)^{2}}\right]
$$

The term $Y_{q}$ indicates the distance of separation between the clusters. This can be achieved by increasing the distance between the cluster centroids. The equation (14) expresses the distance of separation between the clusters. The term $Y_{q}$ defines the average of the minimum of the distance and the median distance between the centroids in the data matrix.

$$
Y_{q}=\frac{1}{2}\left[\min _{i \neq a} F\left(M_{i}^{f}, B_{a}\right)^{2}+\underset{\substack{i \neq a \\ i \neq a}}{\operatorname{dian}} F\left(M_{i}^{f}, B_{a}\right)^{2}\right]
$$

\section{Step 5: Termination}

This is the final step of the CSW-WLIFC algorithm. The condition for the termination of the clustering process is given by the equation (15). The equation 18 expresses that the food image is clustered until all the food items in the image get segmented from each other. The term $\alpha$ indicates the threshold for the termination.

$$
\left\|Y_{a+1}-Y_{a}\right\|<\alpha
$$

At the end of the termination, the food image $I_{f}$ gets segmented into $A$ segments. The equation (16) expresses the segmented image with the proposed CSW-WLIFC algorithm. The term $A_{a}^{f}$ indicates the $a$ th segmented image of the food image $I_{f}$. The value of $a$ varies between $1 \leq a \leq A$.

$$
I_{f}=\left\{A_{1}^{f}, A_{2}^{f}, A_{3}^{f}, \ldots . A_{A}^{f}\right\}
$$

\subsection{Feature extraction}

The segmentation process allows the separation of each food items in the food image. Each food item differs with each other based on their color, shape, and the texture. The feature extraction process allows the extraction of the features such as color, shape, and the texture from the each segmented blocks of the food image. Figure 3 shows the feature extraction process. Each segmented image contains only a single food item, and hence extraction of the features of the food in the image has become easier. The equation (17) expresses the feature extraction of the segmented image $A_{a}^{f}$. The terms $S_{a}^{f}, C_{a}^{f}$ and $Q_{a}^{f}$ expresses the shape, color, and the texture of the segmented image $A_{a}^{f}$.

$$
A_{a}^{f}=\left\{S_{a}^{f}, C_{a}^{f}, Q_{a}^{f}\right\}
$$

3.3a Extraction of the shape features: The shape of the image can be extracted by placing a grid across the segmented image. The size of the grid is represented as $g$. The application of the grid across the image subdivides the segmented image more accurate. The binary value of 1 is substituted in the grid which has the food item. For the other locations of the grid, the binary value of 0 is substituted. Thus, the application of the grid clearly extracts the shape of the food item from the segmented image $A_{a}^{f}$. The shape feature of the food item in the image is represented as $S_{a}^{f}$. The shape feature has the size of $1 \times g$.

3.3b Extraction of the color features: The color feature from the images can be extracted by finding the histogram of the segmented image $I_{a}^{f}$ over the Red, Green, and the Blue channel. The color feature of the segmented image contains the red feature, green feature, and the blue feature. The color feature based on the red channel $H G^{R}\left(A_{a}^{f}\right)$, green channel $H G^{G}\left(A_{a}^{f}\right)$ and the blue channel $H G^{B}\left(A_{a}^{f}\right)$ is shown in equations (18), (19), and (20), respectively.

$$
\begin{array}{ll}
H G_{i}^{R}\left(A_{a}^{f}\right)=\left\{C_{i}^{R} ;\right. & 1 \leq i \leq 255\} \\
H G_{i}^{G}\left(A_{a}^{f}\right)=\left\{C_{i}^{G} ;\right. & 1 \leq i \leq 255\} \\
H G_{i}^{B}\left(A_{a}^{f}\right)=\left\{C_{i}^{B} ; \quad 1 \leq i \leq 255\right\}
\end{array}
$$

Where, $H G_{i}^{R}\left(A_{a}^{f}\right)$ indicates the histogram of the segmented food $A_{a}^{f}$ along the red channel, and $C_{i}^{R}$ indicates the number of pixels in the $i^{\text {th }}$ gray level for the red channel. $H G_{i}^{G}\left(A_{a}^{f}\right)$ indicates the histogram of the segmented food $A_{a}^{f}$ along the green channel, and $C_{i}^{R}$ indicates the number of pixels in the $i^{\text {th }}$ gray level for the green channel. $H G_{i}^{B}\left(A_{a}^{f}\right)$ indicates the histogram of the segmented food $A_{a}^{f}$ along the blue channel, and $C_{i}^{R}$ indicates the number of pixels in the $i^{\text {th }}$ gray level for the blue channel. The equation (21) gives the color feature of the segmented image. The size of the color feature is $3 \times c$. The term $c$ indicates the bin size i.e., $c=255$.

$$
C_{a}^{f}=\sum_{i=1}^{c}\left(H G_{i}^{R}\left(A_{a}^{f}\right)+H G_{i}^{G}\left(A_{a}^{f}\right)+H G_{i}^{B}\left(A_{a}^{f}\right)\right)
$$

3.3c Extraction of the texture features: The texture of the image represents the spatial arrangement of the intensities of the food item in the segmented image. For the simplification process, the segmented food item is represented as 


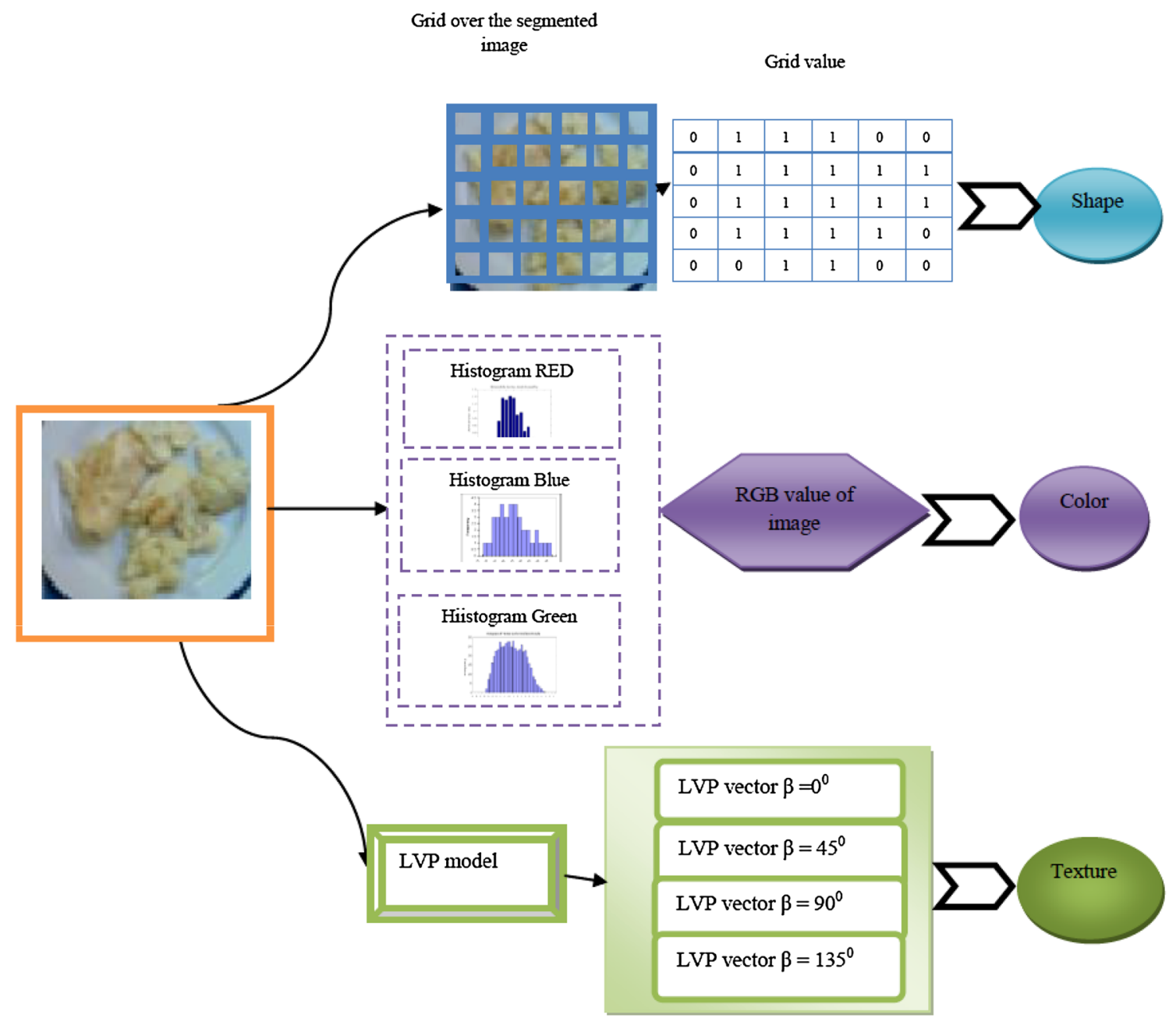

Figure 3. Feature extraction from the segmented image.

$P$. The texture of the segmented image is extracted with the use of the Local Vector Pattern (LVP) [33, 34] model. The LVP model uses the dynamic linear decisive function for extracting the texture feature, and hence the extraction process is more precise. The existing LVP model allows extracting the texture features by constructing the LVP vector. Each pixel in the segmented image is represented as $(i, j)$. Let $P_{i j}$ be the center reference pixel of the segmented image $P$. The LVP model encodes neighboring pixels around the center reference pixel $P_{i j}$ by finding the pair- wise direction of the pixels with the dynamic linear decisive function. The direction value of the vector concerning the center pixel $C_{\beta, O}\left(P_{i j}\right)$ of the segmented image is given in the equation (22),

$$
C_{\beta, O}\left(P_{i j}\right)=\left[P\left(P_{\beta, O}\right)-P\left(P_{i j}\right)\right]
$$

Where. $\beta$ indicates the direction value, $O$ indicates the distance between the reference pixel and the neighbor pixel. The equation (23) expresses the encoding of the LVP vector concerning the center pixel $P_{i j}$.

$$
Q_{H, R, \beta}\left(P_{i j}\right)=\left\{\begin{array}{r}
m_{5}\left[C_{\beta, O}\left(P_{1, R}\right), C_{\beta+45^{0}, O}\left(P_{1, R}\right), C_{\beta, O}\left(P_{i j}\right), C_{\beta+45^{0}, O}\left(P_{i j}\right)\right], \\
m_{5}\left[C_{\beta, O}\left(P_{2, R}\right), C_{\beta+45^{0}, O}\left(P_{2, R}\right), C_{\beta, O}\left(P_{i j}\right), C_{\beta+45^{0}, O}\left(P_{i j}\right)\right], \\
\ldots \ldots \ldots \ldots ., \\
m_{5}\left[C_{\beta, O}\left(P_{H, R}\right), C_{\beta+45^{0}, O}\left(P_{H, R}\right), C_{\beta, O}\left(P_{i j}\right), C_{\beta+45^{0}, O}\left(P_{i j}\right)\right]
\end{array}\right\}
$$


The term $H$ indicates the total number of the neighboring pixels surrounding the reference pixel. The value of the neighboring pixels ranges between $h=0,1,2, \ldots, H$. The term $m_{5}$ indicates the transform ratio and is expressed by the equation (24).

$$
\left.\begin{array}{rl}
m_{5}\left[C_{\beta, O}\left(P_{H, R}\right), C_{\beta+45^{0}, O}\left(P_{H, R}\right), C_{\beta, O}\left(P_{i j}\right), C_{\beta+45^{0}, O}\left(P_{i j}\right)\right] \\
1, \text { if }\left\{\begin{array}{l}
C_{\beta+45^{0}, O}\left(P_{H, R}\right)+ \\
{\left[\frac{C_{\beta+45^{0}, O}\left(P_{i j}\right)}{C_{\beta, O}\left(P_{i j}\right)} * C_{\beta, O}\left(P_{H, R}\right)\right]}
\end{array}\right\} \geq 0 \\
0, \text { else }
\end{array}\right\}
$$

The texture feature of the segmented image is expressed by the equation (25). The LVP vector of the segmented image is the concatenation of the four 8 bit binary patterns formed due to the four directions on the reference pixel. The value of the direction of the vector ranges between $\beta=0^{\circ}, 45^{\circ}, 90^{\circ}, 135^{\circ}$. The term $Q_{H, R, \beta}^{f}\left(P_{i j}\right)$ indicates the LVP of the neighbor pixel concerning the center pixel.

$$
\begin{gathered}
Q_{a}^{f}=Q_{H, R}^{f}\left(P_{i j}\right) \\
Q_{H, R}^{f}\left(P_{i j}\right)=\left\{Q_{H, R, \beta}^{f}\left(P_{i j}\right) ; \beta=0^{\circ}, 45^{\circ}, 90^{\circ}, 135^{\circ}\right\}
\end{gathered}
$$

The LVP feature vector has the size of $4 \times c$. The term $c$ indicates the bin size (i.e., $c=255$ ).

The feature vectors such as shape, color, texture formed from each process are arranged in the feature vector database $D^{F}$. The size of the feature vector database $D^{F}$ is termed as $y$. The equation (27) provides the size of the feature vector database $D^{F}$.

$$
y=[1 \times g+3 \times c+4 \times c]
$$

\subsection{Classification}

3.4a Architecture: The classification is performed to identify the food item from the segmented food image. The extracted features from the segmented image are given to the classifier to identify the food item. The WLM-NN classifier is worked based on the training of the NN with the existing LM [35] and the WOA [36] models. The reason for selecting the WOA and $\mathrm{NN}$ is as follows: When compared to the other optimization algorithms [37], WOA reduces the number of features and improve the classification accuracy simultaneously. The advantages of $\mathrm{NN}$ are, it has the ability to learn and model non-linear and complex relationships, which is really important because in real-life, many of the relationships between inputs and outputs are non-linear as well as complex. Unlike many other prediction techniques, NN does not impose any restrictions on the input variables. Figure 4 shows the block diagram of the
WLM-NN classifier. The existing LM model better utilizes the local minimum function for the training purpose than the global minimum. This allows the LM model to be more robust in nature. The WOA algorithm performs the training of the NN by better utilizing the positions of the image pixels.

The NN network contains three types of layers addressed as i) input layer, ii) hidden layer, and iii) output layer. The total number of the input layers in the $\mathrm{NN}$ is equal to the total feature size of the feature database (i.e., y). The size of the feature vector is expressed in the equation (30). The input layer of the NN is expressed by the equation (28).

$$
U=\left\{U_{1}, U_{2}, U_{3}, \ldots U_{x}, \ldots U_{y}\right\} ; \quad 1 \leq x \leq y
$$

The hidden layers in the NN contain the hidden neurons. The hidden layers of the NN is expressed as follows,

$$
V=\left\{V_{1}, V_{2}, V_{3}, \ldots V_{r}, \ldots V_{s}\right\} ; \quad 1 \leq r \leq s
$$

The hidden layers are formed based on the input layers. The input layer is multiplied with the weight to form the hidden layer. The equation (30) expresses the weight value of each hidden layer. The equation (31) expresses the weight of the hidden layers in the NN.

$$
\begin{gathered}
V_{r}=\frac{1}{y} \sum_{x=1}^{y} k_{x} * U_{x} \\
k=\left\{k_{1}, k_{2}, k_{3}, \ldots, k_{r} \ldots k_{k}\right\}
\end{gathered}
$$

The output layer in the $\mathrm{NN}$ is formed with the hidden layer and the weights. The weights in the NN are modified with the use of the proposed training algorithm.

$$
\begin{gathered}
W=\left\{W_{1}, W_{2}, W_{3}, \ldots, \ldots W_{W}\right\} \\
W=\sum_{r=1}^{s} V_{r} * k_{r}
\end{gathered}
$$

\section{4b Training: A new WLM training algorithm for neural} network:

Step 1:

The initial step in the training phase is the assignment of the learning rate and the delay of the NN. The learning rate is initialized as $\mu=0.1$, and the delay value is $\delta=0.1$. In the first iteration, the weight $k=\left\{k_{1}, k_{2}, k_{3}, \ldots, k_{r} \ldots k_{k}\right\}$ of each neural branch is assigned to the random value.

Step 2:

In this step, the output layer value and the error value are calculated based on the equation (34) and (35). The error value is formed based on the difference of the obtained output of the $\mathrm{NN}$ to the actual value output $l_{x}$. The function $F N N\left(k^{T}, U\right)$ indicates the NN function.

$$
W_{t}^{x}=F N N\left(k^{T}, U\right)
$$






Figure 4. Architecture of the WLM-NN classifier.

$$
\xi_{t}=\sum_{r=1}^{x}\left(W_{t}^{x}-l_{x}\right)
$$

Step 3:

In this step, the weight of the hidden layers of the $\mathrm{NN}$ is modified based on the LM optimization. The weight update by the LM is expressed by the equation (36).

$$
k_{t+1}^{L M}=k_{t}+\Delta k
$$

The change in the weight is obtained with the use of the Jacobian matrix $J_{M}$ and the learning rate $\mu_{t}$ of the NN. The equation (37) expresses the change in weight. The term $I$ indicates the identity matrix. The term $\xi$ indicates the error at the current iteration $t$.

$$
\Delta k=\left[J_{M}^{T} J_{M}+\mu_{t} * I\right] * J_{M}^{T} \xi_{t}
$$

The output layer based on the LM optimization and the modified LM weight is expressed by the equation (38).

$$
W_{t+1}^{x}(L M)=F N N\left(k_{t+1}^{L M}, U\right)
$$

The error rate based on the LM optimization is expressed by the equation (39).

$$
\xi_{t+1}^{L M}=\sum_{r=1}^{x}\left(W_{t+1}^{x}(L M)-l_{x}\right)
$$

Step 4:

In this step, the weight of the hidden layer gets updated based on the Whale optimization. The equation (40) expresses the weight update equation. Where, $\overrightarrow{C_{W H}}$ and $\overrightarrow{A_{W H}}$ indicates the coefficient for the update of the weight.

$$
k_{t+1}^{\text {Whale }}=k_{t}-\overrightarrow{A_{W H}} \cdot \overrightarrow{D_{W H}}
$$

Where,

$$
\overrightarrow{D_{W H}}=\left|\overrightarrow{C_{W H}} \cdot k_{t}-k_{t+1}^{L M}\right|
$$




$$
\begin{gathered}
\overrightarrow{A_{W H}}=2 \overrightarrow{a_{W H}} \quad \cdot \quad \overrightarrow{r_{W H}}-\overrightarrow{a_{W H}} \\
\overrightarrow{C_{W H}}=2 \overrightarrow{a_{W H}} \quad \cdot \quad \overrightarrow{r_{W H}}
\end{gathered}
$$

The value of $a_{W H}$ varies between 0 and 2, and the $r_{W H}$ takes the random value between the $[0,1]$. The output and the error of the $\mathrm{NN}$ based on the Whale optimization are expressed by the equations (44) and (45).

$$
\begin{gathered}
W_{t+1}^{x}(\text { Whale })=F N N\left(k_{t+1}^{\text {Whale }}, n\right) \\
\xi_{t+1}^{\text {Whale }}=\sum_{r=1}^{x}\left(W_{t+1}^{x}(\text { Whale })-l_{x}\right)
\end{gathered}
$$

Step 5:

In this step, the error values obtained from the LM optimization and the Whale optimization are compared using equation (46). If the error value of the LM optimization is small, then the weight update is done based on step 3 . Otherwise, if the error value of the Whale optimization is small, then the weight update is done based on step 4 .

$$
k_{t+1}=\left\{\begin{array}{lc}
k_{t+1}^{L M} ; \quad\left(\xi_{t+1}^{L M} \leq \xi_{t+1}^{\text {Whale }}\right) \\
k_{t+1}^{\text {Whale }} ; \quad \text { otherwise }
\end{array}\right\}
$$

Step 6:

The learning rate of the NN is updated using equation (47).

$$
\mu_{t+1}=\mu_{t} * \delta
$$

Step 7:

The algorithm gets terminated at $t=T$, and the classified output $W$ is obtained from the output layer of the NN.

3.4c Recognition of the segmented food items: In the recognition phase, the feature vectors of the tray image $I_{f}$ of the size $y$ are provided to the trained NN model. The NN model recognizes each food item $A_{a}^{f}$ in the tray image $I_{f}$ with trained weight of $k_{t+1}$.

3.4d Algorithm: Figure 5 shows the WLM-NN classifier algorithm for recognizing the food items from the segmented food images. The NN is trained with the ground truth information $l_{x}$. When the segmented image is provided as the input to the proposed WLM-NN classifier, the food items present in the segmented image are recognized.

\subsection{Estimation of calorie value}

The calorie value of the food items in the image $I_{f}$ can be calculated by finding the calorie value of each segmented food item from the image. The equation (48) expresses the calorie value of the food $I_{f}$.

$$
\operatorname{Calorie}\left(I_{f}\right)=\sum_{a=1}^{A} \operatorname{Calorie}\left(A_{a}^{f}\right)
$$

The equation (49) can calculate the calorie value of the recognized food items in the image $I_{f}$

$$
\begin{aligned}
\operatorname{Calorie}\left(A_{a}^{f}\right)= & \operatorname{Area}\left(A_{a}^{f}\right) \\
& \times \text { Calorie value of one unit square }
\end{aligned}
$$

Where,

$$
\begin{aligned}
\operatorname{Area}\left(A_{a}^{f}\right)= & \text { No of pixels in each segment } \\
& \times \text { Unit Eqiuavalent of one pixel }
\end{aligned}
$$

\section{Results and discussion}

This section analyzes the simulation results of the proposed dietary assessment system with the CSW-WLIFC segmentation and the WLM-NN classifier. The performance of the proposed model is analyzed with the evaluation metrics such as Macro average accuracy (MAA), Mean standard error (MSE) and the Standard accuracy (SA).

\subsection{Experimental set-up}

The simulation of the proposed work is done in the personal computer having the Windows $10 \mathrm{OS}, 4 \mathrm{~GB}$ RAM, and Intel i3 processor. The MATLAB 15 simulator tool provides the simulation results.

a) Dataset description

The food images from the UNIMIB2016 dataset [38] is used for experimentation. The UNIMIB2016 dataset contains 1027 tray images with each food tray containing several food items. There are 73 categories of food items in the tray images. The tray images also contain plates, spoon, fork, and cell phones besides the food items.

\section{b) Evaluation metrics}

The evaluation metrics, such as MAA, MSE, and SA measure the performance of the proposed dietary assessment system with the CSW-WLIFC based segmentation and the WLM-NN classifier. The definition of the evaluation metrics is explained as follows,

1) $M A A$

The MAA defines the average of the true positive of the segmented food item $A_{a}^{f}$ to the number of the times the segmented food item $A_{a}^{f}$ occurs in the tray image $I_{f}$ of the dataset. The equation (51) expresses the mathematical form of the MAA.

$$
M A A=\frac{1}{A} \sum_{a=1}^{A} \frac{T P_{a}^{f}}{N P_{a}^{f}}
$$




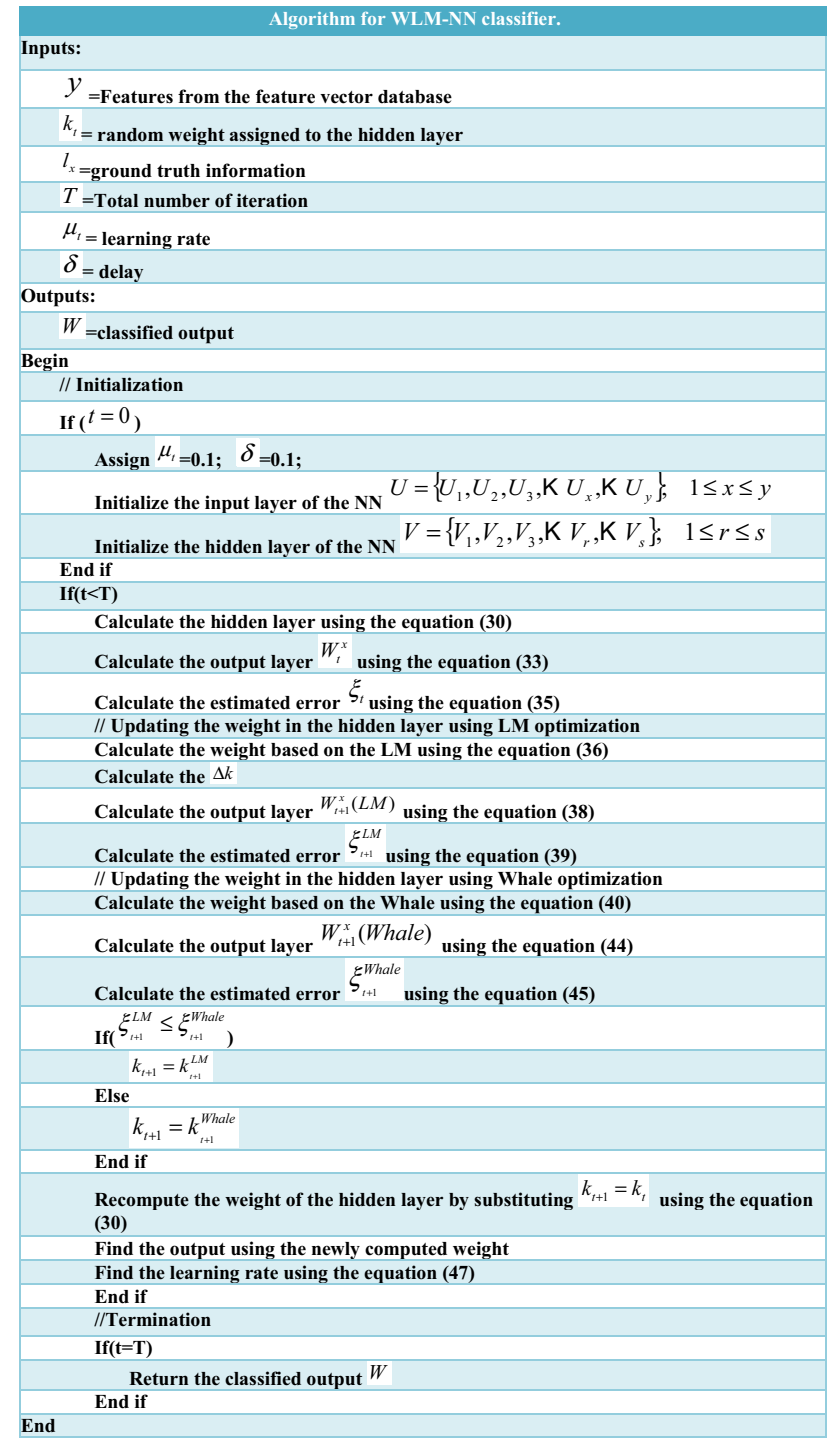

Figure 5. Algorithm for WLM-NN classifier.

where, the term $A$ indicates the total number of food items in the UNIMIB2016 dataset. The term $T P_{a}^{f}$ indicates the true positive of the segmented food item $A_{a}^{f}$ from the tray image $I_{f}$. The term $N P_{a}^{f}$ indicates the number of true positives of the segmented food item $A_{a}^{f}$ from the tray image $I_{f}$.

The tray images in the $I_{f}$ in the dataset vary between $1 \leq f \leq N$.

\section{2) $S A$}

The SA defines the average of the correctly recognized tray images to the total number of tray images in the dataset. The equation (52) expresses the mathematical equation for the SA.

$$
S A=\frac{\sum_{a=1}^{A} T P_{a}^{f}}{\sum_{a=1}^{A} N P_{a}^{f}}
$$

\section{3) $M S E$}

The MSE defines the error performance of the proposed model in terms of the calorie value. The deviation in the calorie value of the food items in the tray image by the proposed model from the ground truth information defines the MSE metric. Equation (53) expresses the MSE metric.

$$
M S E=\frac{1}{A} \sum_{a=1}^{A}\left(\operatorname{Calorie}\left(A_{a}^{f}\right)-l_{f}\right)^{2}
$$

where, the term Calorie $\left(A_{a}^{f}\right)$ indicates the calorie value of the recognized food item by the proposed model from the tray image $I_{f}$, and the term $l_{f}$ indicates the ground truth information of the calorie of the food item in the tray image $I_{f}$. For the better performance, the MSE should be low, and the MAA and SA should be higher.

c) Methods taken for comparison

The performance of the proposed dietary assessment system with the CSW-WLIFC based segmentation and WLM-NN classifier is compared with the existing models, such as WLI-FC with LM-NN, CSW-WLIFC with LM-NN, and WLI-FC with WLM-NN. The evaluation metrics such as SA, MAA, and MSE measure the performance of each model.

\subsection{Experimental results}

Figure 6 shows the original tray images taken from the dataset. The images from the dataset are pre-processed to

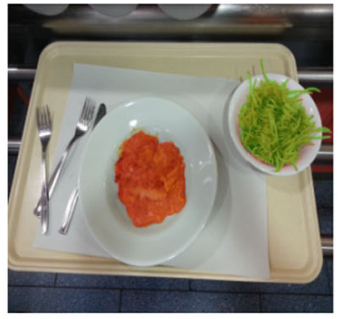

(iii)

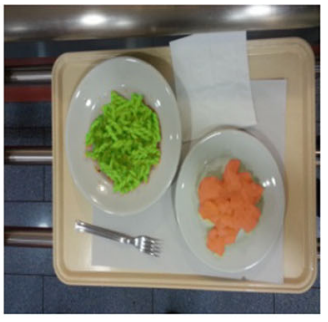

(iv)

Figure 6. Original tray images from the UNIMIB2016 dataset. 




(i)

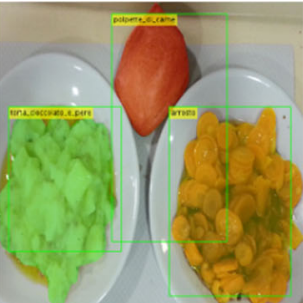

(i)

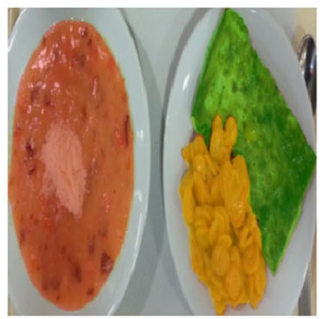

(ii)

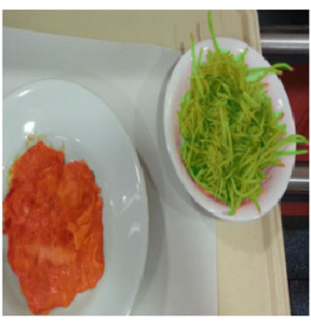

(iii)



(iv)

Figure 7. Preprocessed images.

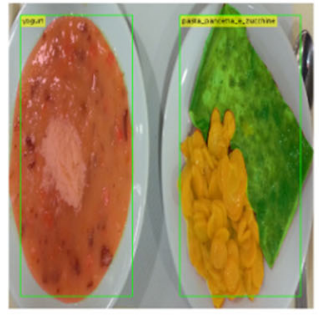

(ii)

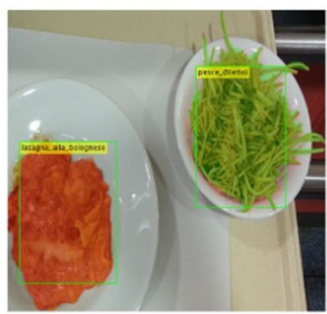

(iii)

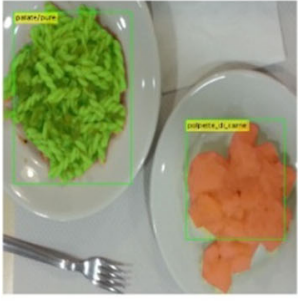

(iv)

Figure 8. Segmentation and the recognition of the food items in the tray image.

recover the region of interest. Figure 7 shows the pre-processed images. The proposed dietary assessment system segments the food items in the tray image with the proposed CSW-WLIFC classifier. The segmented food items are recognized with the proposed WLM-NN classifier. Figure 8 shows the segmented and the recognized food items in each tray image.

\subsection{Performance evaluation}

In this section, the performance of the proposed dietary assessment system with the CSW-WLIFC based segmentation and WLM-NN classifier is analyzed based on the segmentation accuracy. The food items in the tray image are segmented with the proposed CSW-WLIFC based segmentation.

4.3a Performance evaluation based on the segmentation accuracy: The segmentation accuracy of the proposed model should be higher for better performance. Figure 9 shows the performance of the proposed dietary assessment system with the CSW-WLIFC based segmentation based on the segmentation accuracy.

\subsection{Comparative analysis}

The performance of the proposed dietary assessment system with the CSW-WLIFC based segmentation and WLM-NN classifier is compared with the existing models, such as WLI-FC with LM-NN, CSW-WLIFC with LMNN, and WLI-FC with WLM-NN. The existing methods use the metrics such as MSE, MAA, and SA for the performance analysis. Hence these methods are taken for the comparison. Comparative analysis is done by varying the training percentage, hidden layers, and the hidden neurons.

4.4a Comparative analysis based on the MAA: Figure 10 shows the performance of the proposed model against the existing models, such as WLI-FC with LM-NN, CSWWLIFC with LM-NN, and WLI-FC with WLM-NN based on the MAA metric. For the improved performance, the value of the MAA should be higher. When the $75 \%$ of the data from the dataset is trained, the existing WLI-FC with LM-NN, CSW-WLIFC with LM-NN, and WLI-FC with WLM-NN models have the MAA value of $0.9014,0.8802$, and 0.8840 , respectively. The proposed model has the better MAA value of 0.9322 . When the $\mathrm{NN}$ is trained with hidden neurons $=15$, then the existing WLI-FC with LMNN, CSW-WLIFC with LM-NN, and WLI-FC with WLM-NN models have the MAA value of $0.8805,0.8840$, 0.8828 and the proposed model has the MAA value of 0.9495 for the hidden neuron value of 15 . When the NN is trained with five hidden layers, then the existing WLI-FC with LM-NN, CSW-WLIFC with LM-NN, and WLI-FC with WLM-NN model have the MAA value of 0.9021 , 0.9082 , and 0.9190 , respectively. The proposed model outperforms the other existing models with the improved MAA value of 0.9245 . 


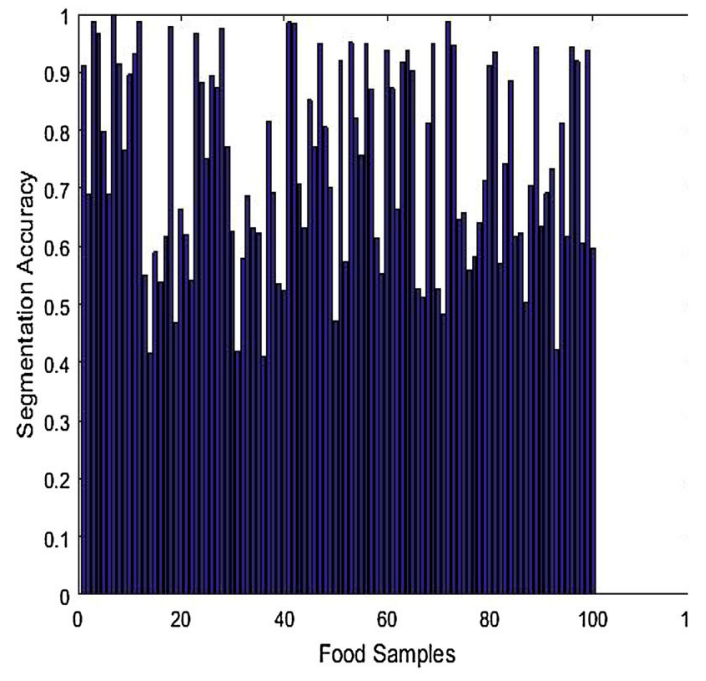

(a) Segmentation accuracy for tray imagesf $=1$ to 100

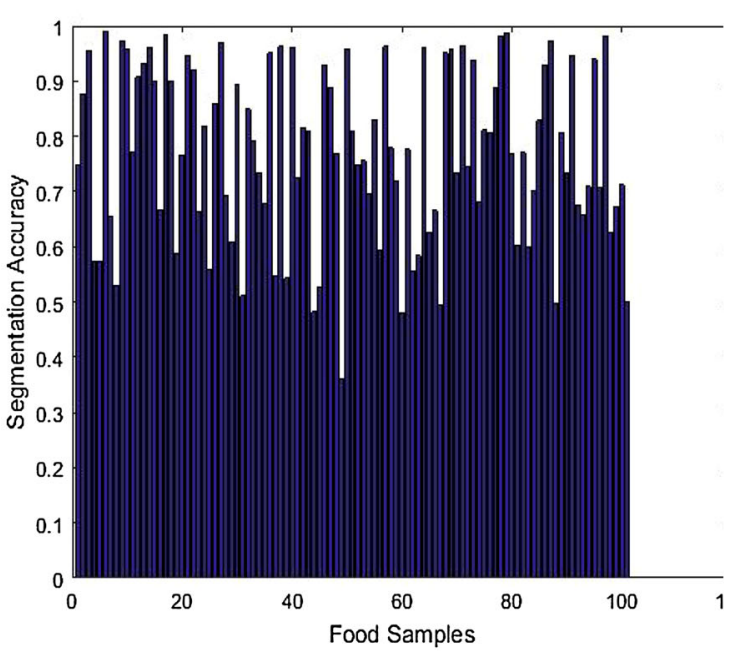

(b) Segmentation accuracy for tray imagesf $=101$ to 200

Figure 9. Performance of the proposed dietary assessment system based on the segmentation accuracy.



(i) Varying training percentage



(ii) Varying the hidden neurons



(iii) Varying the hidden layers

Figure 10. Performance evaluation of the proposed model based on the MAA metric. 


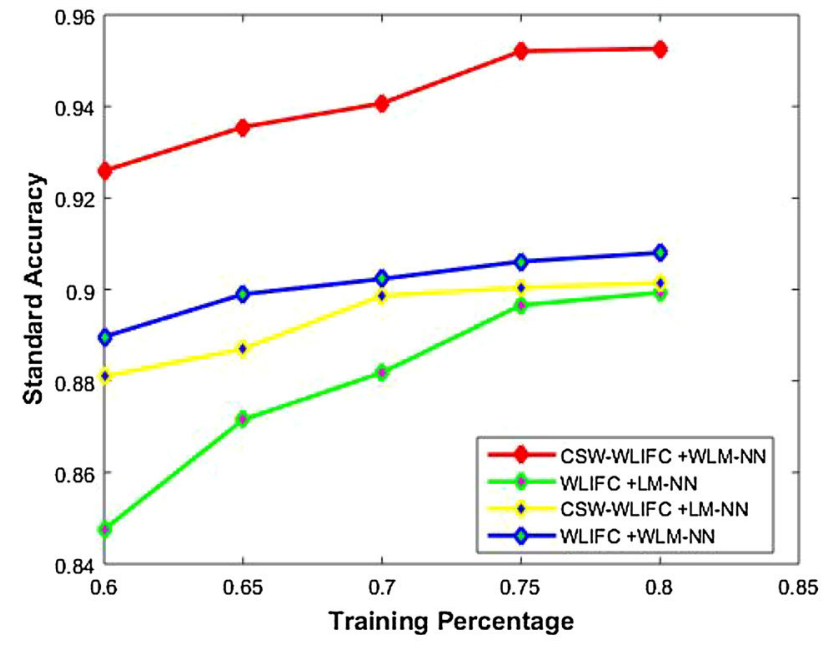

(i) Varying training percentage

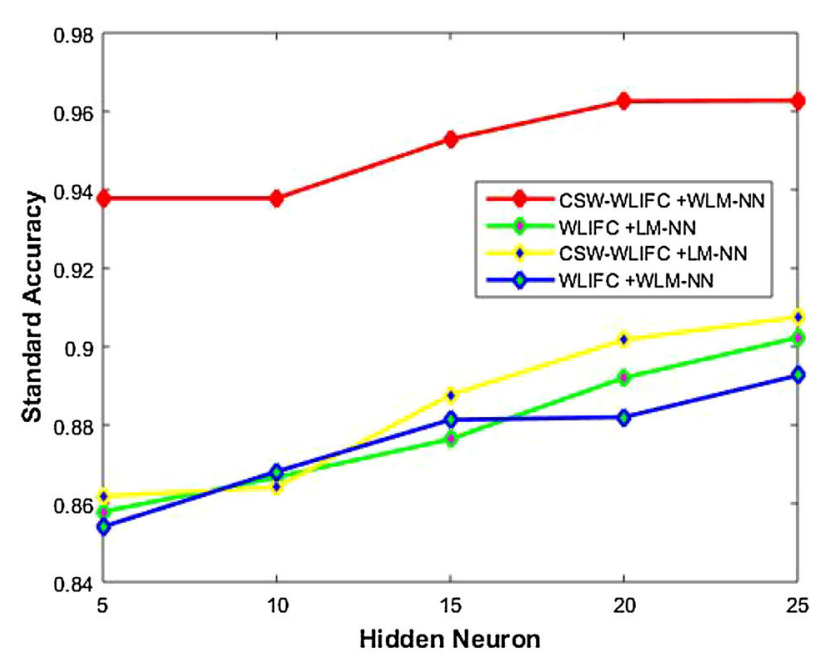

(ii) Varying the hidden neurons

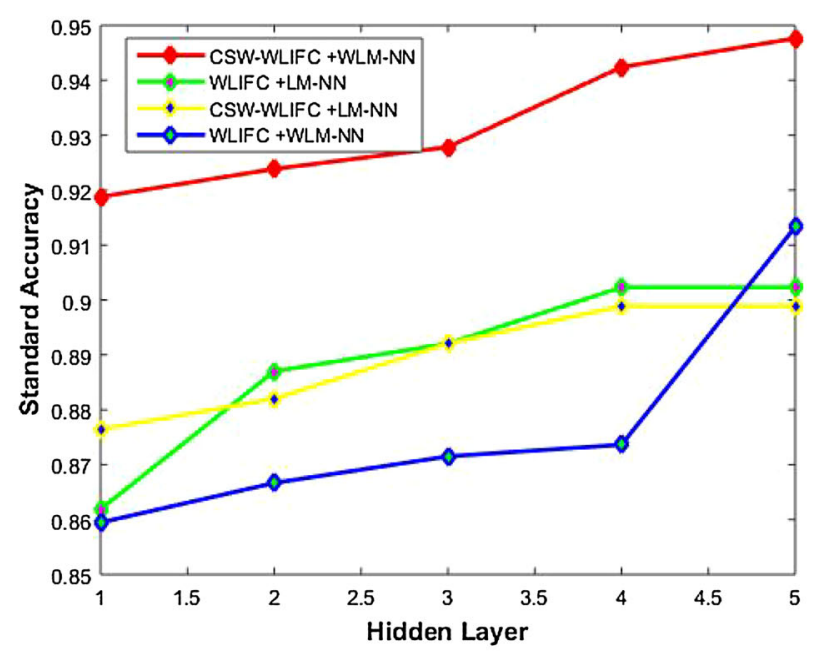

(iii) Varying the hidden layers

Figure 11. Performance evaluation of the proposed model based on the SA metric.

4.4b Comparative analysis based on the SA: Figure 11 shows the performance of the proposed model against the existing models, such as WLI-FC with LM-NN, CSWWLIFC with LM-NN, and WLI-FC with WLM-NN based on the SA metric. For the improved performance, the value of the SA should be higher. When the $75 \%$ of the data from the dataset is trained, the existing WLI-FC with LM-NN, CSW-WLIFC with LM-NN, and WLI-FC with WLM-NN models have the SA value of 0.8965, 0.9003, and 0.9061, respectively. The proposed model has the better SA value of 0.9520 . When the $\mathrm{NN}$ is trained with hidden neurons $=20$, then the existing WLI-FC with LM-NN, CSW-WLIFC with LM-NN, and WLI-FC with WLM-NN models have the SA value of $0.8920,0.9017$, and 0.8820 . The proposed model has the MAA value of 0.9625 for the hidden neuron value of 20 . When the $\mathrm{NN}$ is trained with five hidden layers, then the existing WLI-FC with LM-NN, CSWWLIFC with LM-NN, and WLI-FC with WLM-NN model have the SA value of $0.9022,0.8988$, and 0.9132 , respectively. The proposed model outperforms the other existing models with the improved SA value of 0.9476 .

4.4c Comparative analysis based on the MSE: Figure 12 shows the performance of the proposed model against the existing models such as WLI-FC with LM-NN, CSWWLIFC with LM-NN, and WLI-FC with WLM-NN based on the MSE metric. The MSE metric is analyzed by comparing the calorie value of the recognized food items with the ground truth information. The MSE metric should have low value for better performance. From figures 12(a) and (b) it is concluded that the existing WLI-FC with LM-NN, CSW-WLIFC with LM-NN, and WLI-FC with WLM-NN 


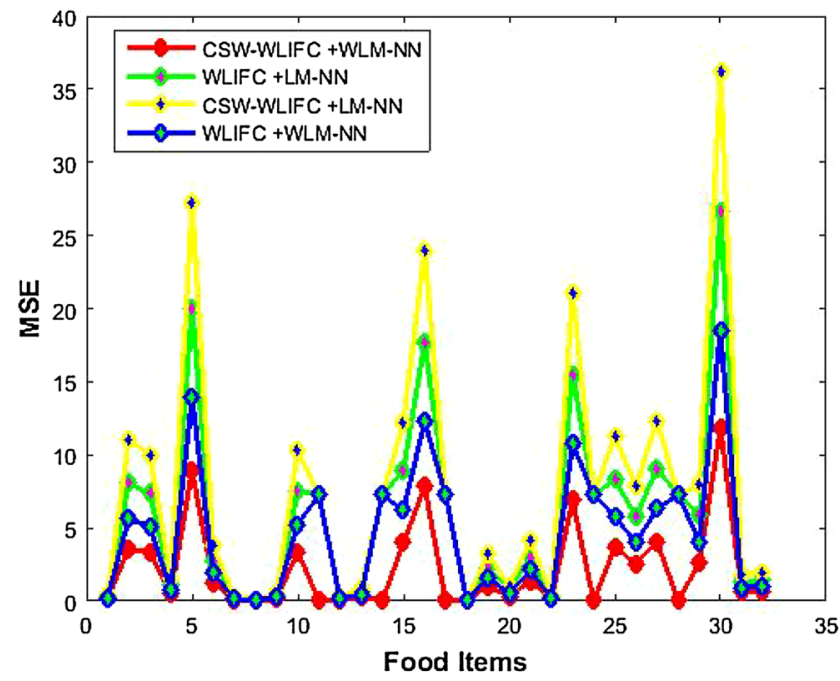

(a) Food items a=1 to 32

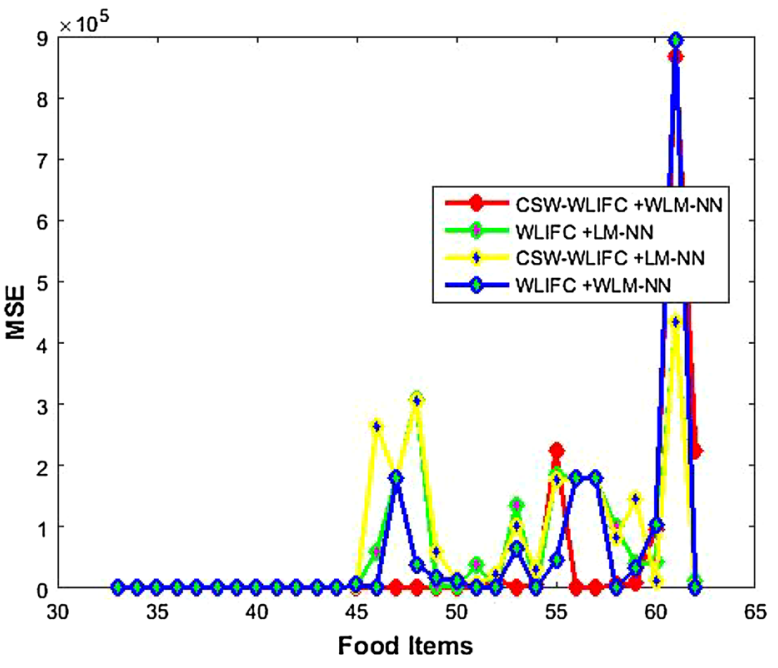

(b) Food items a $=33$ to 62

Figure 12. Performance evaluation of the proposed model based on the MSE metric.

model has the MSE value of $0.3189,0.43269$, and 0.2209 for the food category 1 . The proposed model has the MSE value of 0.1413 for the food category 1 . For the food category 8, the existing WLI-FC with LM-NN, CSW-WLIFC with LM-NN, and WLI-FC with WLM-NN model have the MSE value of $0.0461,0.566$, and 0.0289 . The proposed model has the MSE value of 0.0184 for the food category.

\subsection{Discussion}

The existing models are taken from the paper given in [39]. The various existing models make use of the K-NN and the SVM classifier for recognizing the food items. The existing methods use various approaches for the segmentation. They are Pipelined segmentation and the ground truth segmentation (GT). The segmentation of the food items in the tray images is done by the combination of the approaches: Global approach (G), Local or Patch based approach, Combination of the sum of the posteriors $(G+P)$, and Combination of the product of the posteriors (G X P). The performance of each model is analyzed with the SA, and the MAA metric. The performance of the model is varied based on the feature extraction methods used. The existing models make use of the feature extraction techniques, such as LBP, CEDD, Histogram, Gabor, CNN128, CNN4096, and BoCFR.

Table 2 shows the comparative analysis of the proposed dietary assessment system with the various existing models based on the SA metric. The existing models k-NN + Pipelined $+\mathrm{G}, \mathrm{k}-\mathrm{NN}+$ Pipelined $+\mathrm{P}, \mathrm{k}-\mathrm{NN}+$ Pipelined + $(\mathrm{G}+\mathrm{P})$, and k-NN + Pipelined $+(\mathrm{G} X \mathrm{P})$ has the SA value of $0.728,0.697,0.764,0.763$ when the model uses the CNN4096 feature extraction method. If the existing models use the ground truth approach for the segmentation and the
CNN4096 method for the feature extraction, then the SA is $0.820,0.774,0.885$, and 0.858 for existing models $\mathrm{k}-\mathrm{NN}+$ $\mathrm{GT}+\mathrm{G}, \mathrm{k}-\mathrm{NN}+\mathrm{GT}+\mathrm{P}, \mathrm{k}-\mathrm{NN}+\mathrm{GT}+(\mathrm{G}+\mathrm{P})$, and k-NN $+\mathrm{GT}+(\mathrm{G} X \mathrm{P})$, respectively. The existing models SVM + Pipelined + G, SVM + Pipelined + P, SVM + pipelined + $(\mathrm{G}+\mathrm{P})$, and SVM + pipelined $+(\mathrm{G} \mathrm{X} \mathrm{P})$ has the SA metric of $0.715,0.783,0.798$, and 0.789 , respectively for the CNN4096 feature extraction method. The existing models SVM + GT + G, SVM + GT +P, SVM + GT + $(\mathrm{G}$ $+\mathrm{P})$, and SVM $+\mathrm{GT}+(\mathrm{G} \mathrm{X} \mathrm{P})$ has the SA metric of $0.825,0.857,0.891$, and 0.858 , respectively for the CNN4096 feature extraction method. Here, features such as color, shape, and the texture are extracted from the tray images. The existing models such as WLI-FC + LM-NN, CSW-WLIFC + LM-NN, and WLI-FC + WLM-NN use the proposed feature extraction method and have achieved the SA value of $0.8666,0.9027$, and 0.8882 , respectively. Overall, the proposed CSW-WLIFC based segmentation and WLM-NN classifier model has achieved the best SA metric with the value of 0.9627 and has outperformed the other existing models.

Table 3 shows the comparative analysis of the proposed dietary assessment system with the various existing models based on the MAA metric. The existing models k-NN + Pipelined $+\mathrm{G}, \mathrm{k}-\mathrm{NN}+$ Pipelined $+\mathrm{P}, \mathrm{k}-\mathrm{NN}+$ Pipelined + $(\mathrm{G}+\mathrm{P})$, and k-NN + Pipelined $+(\mathrm{G} \mathrm{X} \mathrm{P})$ has the MAA value of $0.585,0.490,0.561$, and 0.601 . If the existing models use the ground truth approach for the segmentation, then the MAA is $0.652,0.510,0.631$, and 0.685 for existing models $\mathrm{k}-\mathrm{NN}+\mathrm{GT}+\mathrm{G}, \mathrm{k}-\mathrm{NN}+\mathrm{GT}+\mathrm{P}, \mathrm{k}-\mathrm{NN}+\mathrm{GT}+$ $(\mathrm{G}+\mathrm{P})$, and k-NN $+\mathrm{GT}+(\mathrm{G} X \mathrm{P})$, respectively. The existing models SVM + Pipelined $+\mathrm{G}, \mathrm{SVM}+$ Pipelined $+\mathrm{P}, \mathrm{SVM}+$ pipelined $+(\mathrm{G}+\mathrm{P})$, and SVM + pipelined + (G X P) has the MAA metric of $0.546,0.560,0.632$, and 
Table 2. Comparison of the proposed model with the existing models based on SA metric.

\begin{tabular}{|c|c|c|c|c|c|c|c|c|}
\hline \multirow[b]{2}{*}{ Methods } & \multicolumn{8}{|c|}{ SA values for various feature extraction methods } \\
\hline & LBP & CEDD & Hist & Gabor & CNN128 & CNN4096 & BoCFR & Proposed feature \\
\hline $\mathrm{k}-\mathrm{NN}+$ Pipelined $+\mathrm{G}$ & 0.343 & 0.423 & 0.555 & 0.397 & 0.656 & 0.728 & 0.689 & 0.71 \\
\hline $\mathrm{k}-\mathrm{NN}+$ Pipelined $+\mathrm{P}$ & 0.488 & 0.594 & 0.689 & 0.597 & 0.679 & 0.697 & 0.697 & 0.72 \\
\hline $\mathrm{k}-\mathrm{NN}+$ Pipelined $+(\mathrm{G}+\mathrm{P})$ & 0.490 & 0.608 & 0.673 & 0.612 & 0.742 & 0.764 & 0.729 & 0.71 \\
\hline k-NN + Pipelined + (G X P) & 0.436 & 0.477 & 0.637 & 0.461 & 0.714 & 0.763 & 0.716 & 0.75 \\
\hline $\mathrm{k}-\mathrm{NN}+\mathrm{GT}+\mathrm{G}$ & 0.394 & 0.446 & 0.628 & 0.427 & 0.748 & 0.820 & 0.761 & 0.72 \\
\hline $\mathrm{k}-\mathrm{NN}+\mathrm{GT}+\mathrm{P}$ & 0.543 & 0.656 & 0.719 & 0.682 & 0.745 & 0.774 & 0.734 & 0.8 \\
\hline $\mathrm{k}-\mathrm{NN}+\mathrm{GT}+(\mathrm{G}+\mathrm{P})$ & 0.504 & 0.629 & 0.732 & 0.641 & 0.814 & 0.885 & 0.811 & 0.81 \\
\hline $\mathrm{k}-\mathrm{NN}+\mathrm{GT}+(\mathrm{G} \mathrm{X} \mathrm{P})$ & 0.437 & 0.536 & 0.705 & 0.518 & 0.805 & 0.858 & 0.791 & 0.7 \\
\hline SVM + Pipelined $+\mathrm{G}$ & 0.398 & 0.465 & 0.610 & 0.396 & 0.694 & 0.715 & 0.666 & 0.72 \\
\hline SVM + Pipelined $+\mathrm{P}$ & 0.607 & 0.645 & 0.721 & 0.627 & 0.742 & 0.783 & 0.708 & 0.79 \\
\hline SVM + Pipelined $+(\mathrm{G}+\mathrm{P})$ & 0.640 & 0.628 & 0.703 & 0.670 & 0.777 & 0.798 & 0.702 & 0.82 \\
\hline SVM + Pipelined+ (G X P) & 0.489 & 0.555 & 0.612 & 0.529 & 0.746 & 0.789 & 0.689 & 0.75 \\
\hline $\mathrm{SVM}+\mathrm{GT}+\mathrm{G}$ & 0.480 & 0.520 & 0.643 & 0.456 & 0.774 & 0.825 & 0.756 & 0.79 \\
\hline $\mathrm{SVM}+\mathrm{GT}+\mathrm{P}$ & 0.646 & 0.718 & 0.759 & 0.711 & 0.816 & 0.857 & 0.763 & 0.82 \\
\hline $\mathrm{SVM}+\mathrm{GT}+(\mathrm{G}+\mathrm{P})$ & 0.672 & 0.700 & 0.721 & 0.698 & 0.872 & 0.891 & 0.743 & 0.88 \\
\hline $\mathrm{SVM}+\mathrm{GT}+(\mathrm{G} \times \mathrm{P})$ & 0.565 & 0.619 & 0.642 & 0.576 & 0.816 & 0.858 & 0.722 & 0.89 \\
\hline WLI-FC + LM-NN & 0.58 & 0.6 & 0.78 & 0.6 & 0.79 & 0.81 & 0.73 & 0.8666 \\
\hline CSW-WLIFC + LM-NN & 0.59 & 0.69 & 0.77 & 0.65 & 0.77 & 0.82 & 0.8 & 0.9027 \\
\hline WLI-FC + WLM-NN & 0.58 & 0.7 & 0.71 & 0.75 & 0.8 & 0.83 & 0.81 & 0.8882 \\
\hline Proposed approach & 0.6 & 0.712 & 0.798 & 0.78 & 0.82 & 0.89 & 0.89 & 0.9627 \\
\hline
\end{tabular}

0.636, respectively for the CNN4096 feature extraction method. The existing models SVM $+\mathrm{GT}+\mathrm{G}, \mathrm{SVM}+\mathrm{GT}$ $+\mathrm{P}, \mathrm{SVM}+\mathrm{GT}+(\mathrm{G}+\mathrm{P})$, and $\mathrm{SVM}+\mathrm{GT}+(\mathrm{G} \mathrm{X} \mathrm{P})$ has the MAA metric of $0.644,0.575,0.684$, and 0.687 , respectively for the CNN4096 feature extraction method.
The existing models such as WLI-FC + LM-NN, CSWWLIFC + LM-NN, and WLI-FC + WLM-NN use the proposed feature extraction method and have achieved the MAA value of $0.8571,0.8861$, and 0.8828 , respectively. Overall, the proposed CSW-WLIFC based segmentation

Table 3. Comparison of the proposed model with the existing models based on MAA metric.

\begin{tabular}{|c|c|c|c|c|c|c|c|c|}
\hline \multirow[b]{2}{*}{ Methods } & \multicolumn{8}{|c|}{ MAA values for various feature extraction methods } \\
\hline & LBP & CEDD & Hist & Gabor & CNN128 & CNN4096 & BoCFR & Proposed feature \\
\hline $\mathrm{k}-\mathrm{NN}+$ Pipelined $+\mathrm{G}$ & 0.139 & 0.184 & 0.356 & 0.168 & 0.467 & 0.585 & 0.490 & 0.59 \\
\hline $\mathrm{k}-\mathrm{NN}+$ Pipelined $+\mathrm{P}$ & 0.202 & 0.315 & 0.474 & 0.318 & 0.453 & 0.473 & 0.490 & 0.5 \\
\hline $\mathrm{k}-\mathrm{NN}+$ Pipelined $+(\mathrm{G}+\mathrm{P})$ & 0.193 & 0.298 & 0.470 & 0.329 & 0.509 & 0.561 & 0.539 & 0.61 \\
\hline k-NN + Pipelined $+($ G X P $)$ & 0.198 & 0.235 & 0.428 & 0.238 & 0.504 & 0.601 & 0.554 & 0.62 \\
\hline $\mathrm{k}-\mathrm{NN}+\mathrm{GT}+\mathrm{G}$ & 0.171 & 0.219 & 0.380 & 0.192 & 0.555 & 0.652 & 0.559 & 0.61 \\
\hline $\mathrm{k}-\mathrm{NN}+\mathrm{GT}+\mathrm{P}$ & 0.221 & 0.312 & 0.505 & 0.367 & 0.464 & 0.500 & 0.510 & 0.6 \\
\hline $\mathrm{k}-\mathrm{NN}+\mathrm{GT}+(\mathrm{G}+\mathrm{P})$ & 0.210 & 0.313 & 0.493 & 0.377 & 0.586 & 0.631 & 0.571 & 0.641 \\
\hline $\mathrm{k}-\mathrm{NN}+\mathrm{GT}+(\mathrm{G} \mathrm{X} \mathrm{P})$ & 0.222 & 0.273 & 0.457 & 0.291 & 0.611 & 0.685 & 0.614 & 0.69 \\
\hline SVM + Pipelined $+\mathrm{G}$ & 0.185 & 0.215 & 0.346 & 0.203 & 0.479 & 0.546 & 0.449 & 0.59 \\
\hline SVM + Pipelined $+\mathrm{P}$ & 0.332 & 0.356 & 0.483 & 0.377 & 0.496 & 0.560 & 0.479 & 0.6 \\
\hline SVM + Pipelined $+(\mathrm{G}+\mathrm{P})$ & 0.387 & 0.399 & 0.452 & 0.446 & 0.616 & 0.632 & 0.465 & 0.64 \\
\hline SVM + Pipelined $+($ G X P $)$ & 0.281 & 0.354 & 0.367 & 0.322 & 0.626 & 0.636 & 0.442 & 0.712 \\
\hline $\mathrm{SVM}+\mathrm{GT}+\mathrm{G}$ & 0.231 & 0.249 & 0.375 & 0.234 & 0.552 & 0.644 & 0.489 & 0.78 \\
\hline $\mathrm{SVM}+\mathrm{GT}+\mathrm{P}$ & 0.346 & 0.405 & 0.518 & 0.388 & 0.541 & 0.575 & 0.505 & 0.64 \\
\hline $\mathrm{SVM}+\mathrm{GT}+(\mathrm{G}+\mathrm{P})$ & 0.419 & 0.444 & 0.505 & 0.470 & 0.677 & 0.684 & 0.508 & 0.78 \\
\hline $\mathrm{SVM}+\mathrm{GT}+(\mathrm{G} \mathrm{X} \mathrm{P})$ & 0.322 & 0.370 & 0.434 & 0.359 & 0.670 & 0.687 & 0.557 & 0.69 \\
\hline WLI-FC + LM-NN & 0.49 & 0.51 & 0.61 & 0.51 & 0.71 & 0.689 & 0.54 & 0.8571 \\
\hline CSW-WLIFC + LM-NN & 0.47 & 0.542 & 0.62 & 0.56 & 0.78 & 0.658 & 0.64 & 0.8861 \\
\hline WLI-FC + WLM-NN & 0.489 & 0.562 & 0.66 & 0.68 & 0.77 & 0.68 & 0.71 & 0.8828 \\
\hline Proposed approach & 0.51 & 0.578 & 0.69 & 0.69 & 0.76 & 0.81 & 0.82 & 0.9643 \\
\hline
\end{tabular}


and WLM-NN classifier model has achieved the best MAA metric with the value of 0.9643 and has outperformed the other existing models.

\section{Conclusion}

This paper introduces a dietary assessment system based on the proposed CSW-WLIFC based segmentation and WLM-NN classifier. The proposed model segments the food items in the tray with the CSW-WLIFC based segmentation. The CSW-WLIFC based segmentation process utilizes the proposed CSW-based kernel function for the distance calculation. The features such as color, shape, and texture were extracted from the segmented images to form the feature vector database. The WLMNN classifier classifies the segmented food items from the tray image and recognizes each food item. The performance of the proposed dietary assessment system was analyzed with the evaluation metrics such as MAA, MSE, and SA. The food image utilized for the simulation process from the UNIMIB2016 database. The performance of the proposed dietary assessment system is compared with the existing models, such as WLI-FC with LM-NN, CSW-WLIFC with LM-NN, and WLI-FC with WLM-NN. The simulation results show that the proposed model with the CSW-WLIFC based segmentation and WLM-NN classifier has the improved performance than the existing models with the values of $0.999,0.9643$, 0.9627 , and 0.0184 for the segmentation accuracy, MAA, SA, and MSE, respectively.

\section{References}

[1] Martinel N, Piciarelli C and Micheloni C 2016 A supervised extreme learning committee for food recognition. Comput. Vis. Image Underst. 148: 67-86

[2] Liu C, Cao Y, Luo Y, Chen G, Vokkarane V, Ma Y, Chen S and Hou P 2017 A new deep learning-based food recognition system for dietary assessment on an edge computing service infrastructure. IEEE Trans. Serv. Comput. 11(2): 249-261

[3] Foster E and Bradley J 2018 Methodological considerations and future insights for 24-hour dietary recall assessment in children. Nutr. Res. 51: 1-11

[4] Biasse M A D, Bowen D J, Quatromoni P A, Quinn E and Quintiliani L M 2018 Feasibility and acceptability of dietary intake assessment via 24-hour recall and food frequency questionnaire among women with low socioeconomic status. J. Acad. Nutr. Diet. 118(2): 301-307

[5] He H, Kong F and Tan J 2016 DietCam: Multi-view food recognition using a multi-kernel SVM. IEEE J. Biomed. Health Inform. 20(3): 848-855

[6] Rahman M H, Pickering M R, Kerr D, Boushey C J and Delp E J 2012 A new texture feature for improved food recognition accuracy in a mobile phone-based dietary assessment system. In: Proceedings of IEEE International Conference on Multimedia and Expo Workshops (ICMEW), Melbourne, VIC, Australia, pp 418-423

[7] Zhu F, Bosch M, Boushey C J and Edward, Delp J 2015 Multiple hypotheses image segmentation and classification with application to dietary assessment. IEEE J. Biomed. Health Inform. 19(1): 377-388

[8] Velvizhy P, Pavithra and Kannan A 2014 Automatic food recognition system for diabetic patients. In: Proceedings of IEEE Sixth International Conference on Advanced Computing (ICoAC), Chennai, India

[9] Mistura L, Sette S and Mahony C 2013 Modelling framework for the assessment of dietary exposure to added flavouring substances within the FACET (Flavours, Additives, and Food Contact Material Exposure Task) project. Food Chem. Toxicol. 58: 236-241

[10] Jia W, Yue Y, Fernstrom J D, Yao N and Sclabassi R J 2012 Imaged based estimation of food volume using circular referents in dietary assessment. J. Food Eng. 109(1): 76-86

[11] Mogale D G, Kumar S K, Tiwari M K 2016 Two stage Indian food grain supply chain network transportation-allocation model. IFAC PapersOnLine 49(12): 1767-1772

[12] Bag S, Tiwari M K and Chan F T S 2017 Predicting the consumer's purchase intention of durable goods: An attribute-level analysis. J. Bus. Res. Available online 6 December

[13] Kagaya, H, Aizawa K and Ogawa M 2014 Food detection and recognition using convolutional neural network. In: Proceedings of ACM International Conference on Multimedia, Orlando, Florida, USA, pp. 1085-1088

[14] Anthimopoulos M, Dehais J, Diem P and Mougiakakou S 2013 Segmentation and recognition of multi-food meal images for carbohydrate counting. In: Proceedings of IEEE International Conference on Bioinformatics and Bioengineering (BIBE), Chania, Greece, pp. 1-4

[15] Pouladzadeh P, Villalobos G, Almaghrabi R and Shirmohammadi S 2012 A novel SVM based food recognition method for calorie measurement applications. In: Proceedings of IEEE International Conference on Multimedia and Expo Workshops, Melbourne, VIC, Australia, pp. 1-4

[16] Shroff G, Smailagic A and Siewiorek D P 2008 Wearable context-aware food recognition for calorie monitoring, In: Proceedings of 12th IEEE International Symposium on Wearable Computers, Pittsburgh, PA, USA, pp. 119-120

[17] Zhu F, Bosch M, Woo I, Kim S Y, Boushey C J, Ebert D S and Delp E J 2010 The use of mobile devices in aiding dietary assessment and evaluation. IEEE J. Sel. Top. Signal Process. 4(4): 756-766

[18] Kong F and Tan J 2012 DietCam: Automatic dietary assessment with mobile camera phones. Pervasive Mob. Comput. 8(1): 147-163

[19] Bosch M, Zhu F, Khanna N, Carol J, Boushey and Delp E J 2011 Combining global and local features for food identification in dietary assessment. In: Proceedings of IEEE International Conference on Image Processing, Brussels, Belgium, pp. 1789-1792

[20] Kuhad, P, Peddi S V B, Yassine A and Shirmohammadi S 2016 Food calorie measurement using deep learning neural network. In: Proceedings of IEEE Technology Conference on International Instrumentation and Measurement, Taipei, Taiwan, pp. 1-6 
[21] Tammachat N and Pantuwong, N 2014 Calories analysis of food intake using image recognition. In: Proceedings of IEEE International Conference on Information Technology and Electrical Engineering (ICITEE), Yogyakarta, Indonesia, pp. 1-4

[22] Anthimopoulos M M, Gianola L, Scarnato L, Diem P and Mougiakakou Stavroula G 2014 A food recognition system for diabetic patients based on an optimized bag-of-features model. IEEE J. Biomed. Health Inform. 18(4): 1261-1271

[23] LeCun Y, Bottou L, Bengio Y and Haffner P 1998 Gradientbased learning applied to document recognition. Proc. IEEE 86(11): 2278-2324

[24] Mogale D G, Kumar M, Kumar S K and Tiwaria M K 2018 Grain silo location-allocation problem with dwell time for optimization of food grain supply chain network. Transp. Res. Part E Logist. Transp. Rev. 111: 40-69

[25] Mogale D G, Kumar S K and Tiwari M K 2018 An MINLP model to support the movement and storage decisions of the Indian food grain supply chain. Control Eng. Pract. 70: 98-113

[26] Chih-Hung Wu, Ouyang C, Chen L and Lu L 2013 A new fuzzy clustering validity index with a median factor for centroidbased clustering. IEEE Trans. Fuzzy Syst. 23(3): 1-16

[27] Chander S, Vijaya P and Dhyani P 2018 Multi-kernel and dynamic fractional lion optimization algorithm for data clustering. Alex. Eng. J. 57(1): 267-276

[28] Chander S, Vijaya P 2016 MKF-firefly: Hybridization of firefly and multiple kernel-based fuzzy C-means algorithm. Int. J. Adv. Res. Comput. Commun. Eng. 5(7): 213-216

[29] Ramaiah V S and Rao R R 2016 Speaker diarization system using MKMFCC parameterization and WLIfuzzy clustering. Int. J. Speech Technol. 19(4): 945-963

[30] Bonis T and Oudot S 2018 A fuzzy clustering algorithm for the mode-seeking framework. Pattern Recognit. Lett. 102: $37-43$
[31] Liu J, Pham T D, Yan H and Liang Z 2018 Fuzzy mixedprototype clustering algorithm for microarray data analysis. Neurocomputing 276: 42-54

[32] Fahad M, Aadil F, Rehman Z, Khan S, Shah P A, Muhammad K, Loret J L, Wang H, Lee J W, Mehmood I 2018 Grey wolf optimization based clustering algorithm for vehicular ad-hoc networks. Comput. Electr. Eng. Available online 3 February

[33] Satpathy A, Jian X and Eng H 2014 LBP-based edge-texture features for object recognition. IEEE Trans. Image Process. 23(5): 1953-1964

[34] Fan K and Hung T 2014 A novel local pattern descriptor-Local vector pattern in high-order derivative space for face recognition. IEEE Trans. Image Process. 23(7): 2877-2891

[35] Liu H 2010 On the Levenberg-Marquardt training method for feed-forward neural networks. In: Proceedings of IEEE International Conference on Natural Computation, Yantai, China, pp. 1-5

[36] Mirjalili S and Lewis A 2016 The Whale optimization algorithm. Adv. Eng. Softw. 95: 51-67

[37] Maiyar L M and Thakkar J J 2017 A combined tactical and operational deterministic food grain transportation model: Particle swarm based optimization approach. Comput. Ind. Eng. 110: 30-42

[38] UNIMIB2016 database from. http://www.ivl.disco.unimib.it/ activities/food-recognition/. Accessed on 25 March 2017

[39] Ciocca G, Napoletano P and Schettini R 2017 Food recognition for dietary monitoring: A new dataset, experiments, and results. IEEE J. Biomed. and Health Inform. 21(3): $588-598$ 